\title{
Nutritional status and related factors of patients with advanced gastrointestinal cancer
}

\author{
Liyan Zhang ${ }^{1}$, Yuhan $\mathrm{Lu}^{2 *}$ and Yu Fang ${ }^{3}$ \\ ${ }^{1}$ The Key Laboratory of Carcinogenesis and Translational Research (Ministry of Education), Department of Gastrointestinal \\ Oncology, Peking University School of Oncology, Beijing Cancer Hospital and Institute, Beijing, People's Republic of China \\ ${ }^{2}$ Nursing Department, Peking University School of Oncology, Beijing Cancer Hospital and Institute, Beijing 100036, \\ People's Republic of China \\ ${ }^{3}$ Nutrition Section, Beijing Cancer Hospital and Institute, Beijing, People's Republic of China \\ (Submitted 16 July 2013 - Final revision received 24 September 2013 - Accepted 8 October 2013 - First published online 6 January 2014)
}

\section{Abstract}

The scored Patient-Generated Subjective Global Assessment (PG-SGA) is considered to be the most appropriate tool for detecting malnutrition in cancer patients. In particular, malignant tumours derived from the gastrointestinal tract may impair nutrient intake and absorption and cause malnutrition. We carried out a cross-sectional study to assess the nutritional status and related factors of patients with gastrointestinal cancer. Nutritional status was determined using the scored PG-SGA in patients ( $n$ 498) with advanced gastrointestinal cancer admitted to the Gastrointestinal Medical Oncology Unit at Beijing Cancer Hospital between 1 August 2012 and 28 February 2013. The possible related factors including age, sex, hospitalisation frequency and pathology were explored. We found that $98 \%$ of the patients required nutrition intervention and $54 \%$ of the patients required improved nutrition-related symptom management and/or urgent nutritional support (PG-SGA score $\geq 9$ ). Factors related to malnutrition were age $(r 0 \cdot 103, P<0 \cdot 01)$, hospitalisation frequency $(r-0 \cdot 196, P<0 \cdot 01)$ and sex (the prevalence of malnutrition was higher in men than in women $(9 \cdot 88 v \cdot 8 \cdot 54, P<0 \cdot 01)$ ). Patients with rectal cancer had a lower risk of malnutrition than patients with other types of gastrointestinal cancer $(F=35 \cdot 895, P<0 \cdot 01)$. More attention should be paid to the nutritional status of gastrointestinal patients, especially those at a higher risk of malnutrition, such as elderly patients, those hospitalised for the first time, male patients and those with other types of gastrointestinal cancer except rectal cancer. The nutritional status of these patients should be evaluated and they should be given proper nutrition education and nutritional support in a timely manner.

\section{Key words: Patient-Generated Subjective Global Assessment: Gastrointestinal cancer: Malnutrition}

A large number of studies have shown that the incidence of malnutrition is as high as $31-97 \%$ in cancer patients ${ }^{(1-5)}$. Malnutrition can lead to reduced immune function, increased infection rates, prolonged hospitalisation ${ }^{(6)}$, high medical expenditure and increased mortality rates ${ }^{(7,8)}$. Malignant tumours derived from the gastrointestinal tract may impair nutrient intake and absorption and cause malnutrition ${ }^{(9)}$. Therefore, assessing as to how to effectively and accurately determine the nutritional status of gastrointestinal cancer patients, to detect malnutrition and to provide individualised nutritional support is important.

Historically, nutritional status has been evaluated using various objective measures including anthropometric and laboratory measures (e.g. weight change, arm muscle circumference, tricipital skinfold thickness, serum albumin and transferrin and $\mathrm{N}$ balance). Some of these objective measures such as serum albumin are likely to be influenced by many non-nutritional factors $^{(10,11)}$. The evaluation of these measures is often difficult because non-nutritional factors, such as hydration state and disease process, can obscure the effects of actual nutrient deprivation $^{(12)}$. Furthermore, some objective indicators such as serum albumin have long half-lives and thus assessment of changes in the nutritional status over a short period of time is challenging. Moreover, in the clinical setting, methods based on these objective measures are not ideal as they are time consuming and require well-trained staff. In an effort to overcome the limitations of traditional nutritional assessment, an easy-to-use, inexpensive and non-invasive clinical instrument has been developed - the Subjective Global Assessment (SGA).

The SGA is a clinical technique that combines data from subjective and objective aspects of medical history (weight change, dietary intake change, gastrointestinal symptoms and functional capacity changes) and physical examination (subcutaneous fat loss, muscle wasting, ankle or sacral oedema, and ascites) ${ }^{(13)}$.

Abbreviations: PG-SGA, Patient-Generated Subjective Global Assessment; SGA, Subjective Global Assessment.

*Corresponding author: Y. Lu, email lu_yuhan@sina.com 
The scored Patient-Generated SGA (PG-SGA) is a modified version of the SGA and has been specifically developed for patients with cancer ${ }^{(14)}$. Unlike the SGA, which is categorical in nature, the PG-SGA measures nutritional status on a continuous scale, thus allowing for the detection of subtle changes in nutritional status over a short period of time. The higher the PG-SGA score is, the greater the risk of malnutrition is. In addition, unlike the SGA, which must be completed entirely by the health professionals, much of the PG-SGA can be completed by the patients. The scored PG-SGA consists of total PG-SGA score and global assessment. The total PG-SGA score is the sum of scores resulting from seven questions about weight loss, food intake, nutrition impact symptoms, activities and function, disease, metabolic demand and physical examination. Based on the score, four groups are generally recognised: those scoring 0 to 1 points are considered to be normal and do not require intervention; those scoring 2 to 3 points are suspected to have malnutrition or considered to be mildly malnourished and require, along with their family members, nutrition education through a clinician in addition to a pharmacological intervention; those scoring 4 to 8 points require intervention by a dietitian with clinical symptom survey; those scoring $\geq 9$ points are in critical need of improved symptom management and nutrition intervention.

On reviewing the literature, it can be found that although extensive research has been conducted on the validation of the PG-SGA, a relatively small body of research exists regarding the nutritional status of patients with advanced gastrointestinal cancer. To the best of our knowledge, no study has evaluated the nutritional status and related factors using the PG-SGA in advanced gastrointestinal cancer patients. The objectives of the present study were to evaluate the nutritional status and possible relevant factors of patients with advanced (stages III and IV) gastrointestinal cancer using the PG-SGA and provide a basis for nutritional support and individualised care.

\section{Patients and methods}

A cross-sectional study was carried out. The study group consisted of 498 patients with advanced gastrointestinal cancer and aged 24-78 years. The patients admitted to the Gastrointestinal Medical Oncology Unit at Beijing Cancer Hospital between August 2012 and February 2013 were evaluated. The present study was conducted according to the guidelines of the Declaration of Helsinki, and all the protocols involving human subjects were approved by the Ethics Committee of Beijing Cancer Hospital. The assessment of the nutritional status of patients was done within $24 \mathrm{~h}$ of admission to the hospital. Almost all the patients agreed to participate in the study and gave written informed consent. Subjects who had records of weight history for the previous 6 months were selected through purposive sampling. All the patients were confirmed to have gastrointestinal cancer by pathological examination. Data on a wide range of variables including demographic factors (age, sex, hospitalisation frequency and pathology) and nutritional status (PG-SGA score, serum albumin, weight loss, BMI, dietary intake, nutrition-related symptoms, activities and functions, subcutaneous fat loss, stress and body fluid) were collected.

\section{Nutritional status assessment}

Nutritional history (changes in body weight, dietary intake and functional capacity and gastrointestinal symptoms and infections) and clinical status were determined using the PG-SGA form. The sum of scores obtained in each domain of the PG-SGA was used to determine the total PG-SGA score. After consultation, the dietitians ranked the nutritional status of the patients as follows: 0 to 1 - nutritional support not required and treatment in the future based on routine re-evaluation; 2 to 3 - dietary guidance for patients and their families by a nutritionist, with assistance from nurses or other health care professionals based on symptom investigation and laboratory examination; 4 to 8 - nutritional support provided by a dietitian with assistance from nurses or physicians according to the symptom questionnaire; $\geq 9$ - urgent need for improved symptom management and/or nutritional support. The American Dietetic Association recommends the PG-SGA for rating the nutritional status of cancer patients ${ }^{(3)}$.

\section{Domains of the Patient-Generated Subjective Global Assessment}

The PG-SGA has seven domains: weight loss; food intake; nutrition impact symptoms; activities and function; disease and its relation to nutritional requirements; metabolic demand; nutritionrelated physical examination. Each of these domains is given 0 to 4 points depending on the impact of the component on nutritional status. The sum of scores obtained in each domain provides a guideline as to the level of nutrition and pharmacological intervention required for each patient. Each of the domains is described below.

Weight loss. This domain includes data on the current body weight $(\mathrm{kg})$ and height $(\mathrm{m})$ of the patients. It also includes data on weight in the past 6 months and past 1 month. The domain documents whether the weight of the patients has decreased, remained unchanged or increased in the past 2 weeks. The obtained current body weight value is used to determine the level of weight change in the past 2 weeks and is scored from 0 to 1 . Patients with weight loss in the previous 2 weeks are given a score of 1 and those without any change in weight status are given a score of 0 .

Food intake. This domain rates the current food intake of the patients compared with that in the past month. It establishes whether food intake is unchanged, more than usual or less than usual. The domain determines whether the patients are currently consuming normal or less than normal amounts of food, little amounts of solid food, only liquids, only nutritional supplements, or very little of anything or given only tube feedings or parenteral nutrition.

Nutrition impact symptoms. This domain contains a checklist of gastrointestinal problems that interfere with the adequate dietary intake of the patients during the past 2 weeks. These include nausea, oral sores/ulcers, painful swallowing, dysphagia, appetite loss, diarrhoea, being bothered by smell, feeling full quickly and fatigue. Scores for these items range 
from 0 to 3 depending on their impact on dietary intake. A score of 0 indicates no problem while eating, whereas a score of 3 indicates the presence of problems with a severe impact on dietary intake. Vomiting, anorexia, diarrhoea and pain are given the highest scores.

Activities and function. This domain rates the activities of the patients over the past month based on the following options: normal with no limitations; not normal but able to perform fairly normal activities; not feeling up to most things but in bed or chair less than half the day; able to perform little activity and spent most of the day in bed or chair; pretty much bedridden, rarely out of bed.

Disease and its relation to nutritional requirements. This domain contains a checklist of all the relevant diagnoses, and others not in the checklist are required to be specified. A maximum score of 1 is given to each diagnosis.

Metabolic demand. This domain scores metabolic stress by determining the number of variables known to increase protein and energy requirements. It specifically scores the intensity of fever, duration of fever and corticosteroids used.

Nutrition-related physical examination. This domain includes a subjective evaluation of two aspects of body composition: fat and fluid status. Each aspect of the examination is rated for the degree of deficit, with muscle defect affecting the score more than fat deficit. Tricipital skinfold and fat overlying the lower ribs are examined to determine the level of fat loss. Since the assessment is subjective, each aspect of the examination is rated for a global degree of deficit. Patients without any deficit are given a score of 0 , while those with mild, moderate and severe deficits are given scores of 1, 2 and 3, respectively.

Anthropometric assessment. The current body weight (kg) and height $(\mathrm{m})$ of the patients were measured following the WHO standard procedures. Weight was measured using the Pu Heng RGZ-120-RT scale (Jiangsu PU Heng manufacturing company) and recorded to the nearest $0 \cdot 1 \mathrm{~kg}$. Measurements were repeated twice, and the average was used as the final value for each subject. Height was measured using the $\mathrm{Pu}$ Heng RGZ-120-RT meter (Jiangsu PU Heng manufacturing company) and was recorded to the nearest $0.1 \mathrm{~cm}$. The measured body weight and height were used to calculate the BMI of the subjects by dividing body weight by the square of height $\left(\mathrm{kg} / \mathrm{m}^{2}\right)$. The current body weight was also used to determine the level of weight change in the past 2 weeks and was scored from 0 to 1 as indicated in the PG-SGA. Subjects who reported weight loss in the previous 2 weeks were given a score of 1 , while those with no change in weight status were given a score of 0 . Weight recorded over the last 6 months was retrieved from each participant's file and used to compute the percentage of weight change as follows:

$$
\begin{aligned}
& \% \text { Weight change } \\
& =\frac{\text { usual body weight }- \text { actual body weight }}{\text { usual body weight }} \times 100 \text {. }
\end{aligned}
$$

To determine the weight loss score, data on weight in the previous 1 month were used, while those on weight in the previous 6 months were used only if those of the previous 1 month were not available. Weight loss of $10 \%$ or greater in 1 month was given a score of 4 , while that of $0-1.9 \%$ was given a score of 0 . Weight loss of $20 \%$ or greater in 6 months was given a score of 4 , while that of $0-1.9 \%$ was given a score of 0 .

\section{Statistical analysis}

Statistical analysis was carried out using SPSS version 19 (SPSS Institute, Inc.). Anthropometric and PG-SGA scores are presented as descriptive statistics (means, standard deviations and frequencies). The degree of relationship among these factors and PG-SGA scores was statistically evaluated using the $t$ test (different sex), ANOVA test (different pathology) and correlation analyses (age and hospitalisation frequency). Statistical significance is reported at the $P<0.05$ level (twotailed). The present cross-sectional study was carried out in subjects who had advanced gastrointestinal cancer and were admitted to the Gastrointestinal Medical Oncology Unit at Beijing Cancer Hospital from August 2012 to February 2013.

\section{Results}

A total of 498 patients were hospitalised at the Gastrointestinal Medical Oncology Unit from August 2012 to February 2013, of which 352 were males and 146 were females aged 24-78 years, with a mean age of 56.35 (SD 10.76) years. According to the location of the tumour, there were $106(18.0 \%)$ patients with oesophageal cancer, $217(36 \cdot 8 \%)$ with gastric cancer, fifty-six $(9.5 \%)$ with colon cancer, eighty-five (14.4\%) with rectal cancer, sixteen $(2 \cdot 7 \%)$ with pancreatic and bile duct cancer, and eighteen (3.1\%) with other cancers. All were confirmed by pathological diagnosis. Patients without heart, lung, kidney, liver and infectious diseases were enrolled in the study.

The nutritional status of the study group is summarised in Table 1. The PG-SGA scores of all the patients ranged from 1 to 22 , with the mean value being 9.48 (SD 4.31). Based on the scores, only $2 \%$ of the patients did not need nutritional intervention (scores 0 to 1 ), about one-third (32.5\%) needed nutritional support and over half of them (57.4\%) needed nutrition-related symptom management and (or) nutritional support urgently (score $\geq 9$ ).

The prevalence of weight loss among the subjects was $58.6 \%$, with an average of $2.94 \%$, including weight loss up to $28 \%$ in 3 months in one patient and severe weight loss in $9 \%$ of the patients (weight loss of more than $10 \%$ in

Table 1. Patient-Generated Subjective Global Assessment classification ( $n$ 498)

\begin{tabular}{lcc}
\hline Classification (score) & Cases $(n)$ & $\%$ \\
\hline $\begin{array}{l}\text { Without intervention } \\
(0 \text { to } 1)\end{array}$ & 10 & $2 \cdot 0$ \\
$\begin{array}{l}\text { Need health education } \\
(2 \text { to } 3)\end{array}$ & 40 & $8 \cdot 0$ \\
$\begin{array}{l}\text { Need nutritional support } \\
\quad(4 \text { to } 8)\end{array}$ & 162 & 32.5 \\
$\begin{array}{l}\text { Need symptom management } \\
\text { and/or nutritional support }(\geq 9)\end{array}$ & 286 & $57 \cdot 4$ \\
\hline
\end{tabular}

6 months were used only if those of the previous 1 month were 
1 month or more than $20 \%$ in 6 months), and weight loss continued to occur in $72.7 \%$ of the patients. Nearly, four-fifths $(79.7 \%)$ of the patients had difficulty in eating food, including $19.7 \%$ who could consume only liquid food and $4 \%$ who could not eat anything. Furthermore, $80.7 \%$ of the subjects had nutrition impact symptoms, including nausea or fullness (27.7\%), choking (14.3\%), appetite loss, vomiting and diarrhoea $(38.8 \%)$. Of the subjects, $77.5 \%$ had impaired function and ability. Among them, $16.9 \%$ could barely sit or lie in bed all day. In addition, $67 \cdot 7 \%$ of the patients reported fat loss, with the condition being severe and in $30 \%$. Additionally, $2.4 \%$ of the patients experienced moderate-to-severe stress (high fever) and $4 \%$ of the patients had pleural effusion or ascites. Table 2 summarises the characteristics of the patient cohort in detail.

Table 3 summarises the relationship between PG-SGA scores and possible related factors. Correlation analyses indicated the presence of a weak association between PG-SGA scores and two factors: age $(r 0 \cdot 013, P<0 \cdot 01)$ and hospitalisation frequency

Table 2. Patient-Generated Subjective Global Assessment contents (n 498)

\begin{tabular}{|c|c|c|}
\hline & Cases $(n)$ & $\%$ \\
\hline \multicolumn{3}{|l|}{ Weight loss ratio $(\%)^{*}$} \\
\hline 0 to 1.9 & 206 & 41.4 \\
\hline 2 to 2.9 in 1 month or 2 to 5.9 in 6 months & 48 & $9 \cdot 6$ \\
\hline 3 to 4.9 in 1 month or 6 to 9.9 in 6 months & 96 & $19 \cdot 3$ \\
\hline $\begin{array}{l}5 \text { to } 9.9 \text { in } 1 \text { month or } 10 \text { to } 19.9 \text { in } \\
6 \text { months }\end{array}$ & 103 & $20 \cdot 7$ \\
\hline$\geq 10$ in 1 month or $\geq 20$ in 6 months & 45 & $9 \cdot 0$ \\
\hline \multicolumn{3}{|l|}{ Body-weight loss in the recent 2 weeks } \\
\hline Yes & 362 & $72 \cdot 7$ \\
\hline No & 136 & $27 \cdot 3$ \\
\hline \multicolumn{3}{|l|}{ Food intake } \\
\hline Normal & 101 & $20 \cdot 3$ \\
\hline Reduced food intake & 161 & $32 \cdot 3$ \\
\hline Pap & 118 & $23 \cdot 7$ \\
\hline Liquid food & 98 & $19 \cdot 7$ \\
\hline Could not consume food & 20 & 4.0 \\
\hline \multicolumn{3}{|l|}{ Nutrition impact symptoms } \\
\hline No & 96 & $19 \cdot 3$ \\
\hline Nausea and fullness & 138 & $27 \cdot 7$ \\
\hline Choking & 71 & $14 \cdot 3$ \\
\hline Appetite loss/vomiting/diarrhoea & 193 & $38 \cdot 8$ \\
\hline \multicolumn{3}{|l|}{ Functional capacity } \\
\hline Normal & 117 & 23.5 \\
\hline Minor activities & 228 & $45 \cdot 8$ \\
\hline Lying or sitting for less than half a day & 69 & $13 \cdot 9$ \\
\hline Lying or sitting for more than half a day & 84 & $16 \cdot 9$ \\
\hline \multicolumn{3}{|l|}{ Loss of fat (tricipital skinfold thickness) } \\
\hline No & 166 & $33 \cdot 3$ \\
\hline Mild & 55 & $11 \cdot 0$ \\
\hline Moderate & 133 & $26 \cdot 7$ \\
\hline Severe & 144 & 28.9 \\
\hline \multicolumn{3}{|l|}{ Stress } \\
\hline No & 486 & $97 \cdot 6$ \\
\hline Moderate & 3 & 0.6 \\
\hline Severe & 9 & 1.8 \\
\hline \multicolumn{3}{|l|}{ Body fluids (pleural effusion and ascites) } \\
\hline No & 478 & $96 \cdot 0$ \\
\hline Mild & 1 & 0.2 \\
\hline Moderate & 5 & 1.0 \\
\hline Severe & 14 & $2 \cdot 8$ \\
\hline
\end{tabular}

* Ranged from -7.14 to $28 \%$, with a mean of 2.94 (SD 4.68 ). $(r-0 \cdot 196, P<0 \cdot 01)$. The $t$ test indicated that the scores for males were higher than those for females (9.88v. 8.54, $P<0.01)$. Comparison of nutritional status in subjects with cancer of different pathological types showed that the score of those with rectal cancer was lower than that of those with other cancers $(F=35 \cdot 895, P<0 \cdot 000)$.

\section{Discussion}

American cancer nutrition researchers suggest that about $40 \%$ of cancer patients actually die from malnutrition, rather than from cancer and aggressive treatment ${ }^{(15)}$. The results of the present study (Table 1) indicate that only $2 \%$ of patients with gastrointestinal cancer do not need nutritional intervention and $57.4 \%$ of the patients need nutrition-related symptom management and/or nutritional support urgently. Our finding is consistent with Segura's ${ }^{(2)}$ report. It has been indicated that malnutrition in gastrointestinal cancer patients is prevalent and that these patients require attention and timely nutrition education and professional support.

Data summarised in Table 2 indicate that gastrointestinal cancer patients experienced various degrees of weight loss, with an average of $2.94 \%$. Weight loss was up to $28 \%$ in 3 months, including severe weight loss in $9 \%$ of the patients (weight loss of more than $10 \%$ in 1 month or more than $20 \%$ in 6 months), and weight loss continued to occur in $72.7 \%$ of the patients. Moreover, $67.7 \%$ of the patients had fat loss, with it becoming severe in $30 \%$. Besides that, $2.4 \%$ of the patients experienced moderate-to-severe stress (high fever) and $4 \%$ had pleural effusion or ascites. The main causes of malnutrition in patients with gastrointestinal cancer are the abnormal increased metabolism induced by the tumour, difficulty in eating and digestion, and side effects of anticancer therapy ${ }^{(16-18)}$ such as nausea, vomiting, fatigue and pain. The present study indicates that nearly $80 \%$ of the patients had difficulty in eating and $4 \%$ almost could not eat anything. At the same time, $80.7 \%$ of the patients had nutritional intakerelated symptoms, with the most common symptoms being appetite loss or vomiting and diarrhoea. Malnutrition led to various degrees of decline in social activity and function in $77.5 \%$ of the patients, among them $16.9 \%$ could just sit or lie in bed all day. There is an urgent need to improve the nutritional status of gastrointestinal cancer patients.

Factors related to malnutrition assessed by the PG-SGA were patients' age, hospitalisation frequency, sex and tumour location (Table 3). The age of the patients and the PG-SGA score were positively correlated, which means that elderly patients would be more likely to have malnutrition. The physiological functions of major organs in elderly may deteriorate with increasing age and the compensatory ability may also decrease. Furthermore, accompanying chronic diseases such as diabetes may exacerbate physical conditions. Thus, more attention should be paid to these elderly patients. The severity of malnutrition decreased with the prolongation of hospitalisation. This may be correlated with the termination of treatment, the diminishing or shrinkage of the tumour, and the improvement in food intake. The study also demonstrates that male patients would be more likely to have malnutrition. 
Table 3. Patient-Generated Subjective Global Assessment (PG-SGA) scores for age, hospitalisation frequency, sex and diagnosis ( $n$ 498)

(Means values and standard deviations)

\begin{tabular}{lcccr}
\hline & \multicolumn{2}{c}{ PG-SGA score } & & \\
\cline { 2 - 3 } & Mean & SD & Statistical value & $P$ \\
\hline Age & & & $R 0.103$ & 0.002 \\
Hospitalisation frequency & & & $R-0.196$ & 0.000 \\
Sex & 9.88 & 4.15 & $t=3.169$ & 0.002 \\
$\quad$ Male & 8.54 & 4.56 & & \\
$\quad$ Female & & & & 0.000 \\
Diagnosis & $11.07^{\star}$ & 4.03 & & \\
$\quad$ Oesophageal cancer & 10.35 & 3.79 & & \\
Gastric cancer & $9.54 \dagger \dagger$ & 3.50 & & \\
$\quad$ Colon cancer & 4.67 & 2.90 & $F=35.895$ & \\
$\quad$ Rectal cancer & 10.94 & 4.84 & & \\
$\quad$ Pancreatic and bile duct cancer & 10.94 & 3.65 & & \\
$\quad$ Other cancers & & & \\
\hline
\end{tabular}

${ }^{*}$ Mean value was significantly different from that of colon cancer $(P<0.05)$.

†† Mean value was significantly different from that of all other types of cancer $(P<0.01)$.

The reason for this is unclear, but one possible explanation is the relatively low fat proportion in males, which could be easily exhaustive. So, more attention should be paid to male patients, and appropriate nutrition education and professional support should be given as early as possible. Additionally, the incidence of malnutrition is also associated with the location of tumours. Patients with rectal cancer are at a lower risk of becoming malnourished. Our observation is similar to Segura's ${ }^{(2)}$ finding. As rectal cancer occurs in the lower gastrointestinal tract, tumour in this region does not affect the intake and absorption of nutrients. This is quite different from oesophagus cancer, which is a more possible cause of malnutrition.

The present study is the first to explore the possible factors of malnutrition in China. It aimed to draw more attention to the nutritional status of patients with gastrointestinal cancer, especially those at a higher risk of malnutrition, such as elderly patients, those hospitalised for the first time, male patients and those with other types of gastrointestinal cancer except rectal cancer. Patients with a high risk of malnutrition should be evaluated and given nutrition education and necessary nutritional support in time. The improvement of the nutritional status of patients has beneficial effects on their quality of life.

\section{Conclusion}

The present study is the first to evaluate the nutritional status of gastrointestinal cancer patients using the PG-SGA with a large sample size in China. The study demonstrates that $98 \%$ of gastrointestinal cancer patients need nutritional support and intervention and that more than half the patients urgently need nutritional interventions and nutrition-related symptom management at the same time. More attention should be paid to the nutritional status of patients with gastrointestinal cancer, especially those at a higher risk of malnutrition, such as elderly patients, those hospitalised for the first time, male patients and those with other types of gastrointestinal cancer except rectal cancer. These high-risk patients should be evaluated and given nutrition education and necessary nutritional support in time.

The present study has several limitations. First, this is a single-centre study. Further multi-centre research should be carried out to verify the findings of the present study. Second, no intervention was given to the malnourished subjects. This should be done in the future to determine whether a change in the nutritional status could improve the quality of life of gastrointestinal cancer patients.

\section{Acknowledgements}

The present study did not receive any funding support.

The authors' contributions are as follows: L. Z. contributed to the design and conduct of the study, analysis of the samples and data, interpretation of the findings and preparation of the manuscript; Y. L. provided valuable guidance regarding study design and writing process; Y. F. obtained the PG-SGA form and gave directions on how to use it.

None of the authors has any conflicts of interest to declare.

\section{References}

1. Huhmann MB \& Cunningham RS (2005) Importance of nutritional screening in treatment of cancer-related weight loss. Lancet Oncol 6, 334-343.

2. Segura A, Pardo J \& Jara C (2005) An epidemiological evaluation of the prevalence of malnutrition in Spanish patients with locally advanced or metastatic cancer. Clin Nutr $\mathbf{2 4}$, 801-814.

3. Abe Vicente M, Barão K, Silva TD, et al. (2013) What are the most effective methods for assessment of nutritional status in outpatients with gastric and colorectal cancer? Nutr Hosp $\mathbf{2 8}$, 585-591.

4. Malihi Z, Kandiah M, Chan YM, et al. (2013) Nutritional status and quality of life in patients with acute leukaemia prior to and after induction chemotherapy in three hospitals in Tehran, Iran: a prospective study. J Hum Nutr Diet 26 , Suppl. 1, 123-131.

5. Gabrielson DK, Scaffidi D, Leung E, et al. (2013) Use of an abridged scored Patient-Generated Subjective Global 
Assessment (abPG-SGA) as a nutritional screening tool for cancer patients in an outpatient setting. Nutr Cancer $\mathbf{6 5}$, 234-239.

6. Gupta D, Vashi PG, Lammersfeld CA, et al. (2011) Role of nutritional status in predicting the length of stay in cancer: a systematic review of the epidemiological literature. Ann Nutr Metab. 59, 96-106.

7. Hill A, Kiss N, Hodgson B, et al. (2011) Associations between nutritional status, weight loss, radiotherapy treatment toxicity and treatment outcomes in gastrointestinal cancer patients. Clin Nutr 30, 92-98.

8. Laky B, Janda M, Kondalsamy-Chennakesavan S, et al. (2010) Pretreatment malnutrition and quality of life - association with prolonged length of hospital stay among patients with gynecological cancer: a cohort study. BMC Cancer. 10, 232.

9. Dias do Prado C \& Alvares Duarte Bonini Campos J (2013) Nutritional status of patients with gastrointestinal cancer receiving care in a public hospital; 2010-2011. Nutr Hosp 28, 405-411.

10. Bauer J, Capra S \& Ferguson M (2002) Use of the scored Patient-Generated Subjective Global Assessment (PG-SGA) as a nutrition assessment tool in patients with cancer. Eur J Clin Nutr 56, 779-785.
11. Leuenberger M, Kurmann S \& Stanga Z (2010) Nutritional screening tools in daily clinical practice: the focus on cancer. Support Care Cancer 18, Suppl. 2, S17-S27.

12. Detsky AS, Baker JP, Mendelson RA, et al. (1984) Evaluating the accuracy of nutritional assessment techniques applied to hospitalized patients: methodology and comparisons. J Parenter Enteral Nutr 8, 153-159.

13. Detsky AS, McLaughlin JR, Baker JP, et al. (1987) What is subjective global assessment of nutritional status? J Parenter Enteral Nutr 11, 8-13.

14. Ottery FD (1996) Definition of standardized nutritional assessment and interventional pathways in oncology. Nutrition 12, S15-S19.

15. Li J (1993) Clinical Enteral and Parenteral Nutrition, pp. 96-100. Beijing: People's Medical Publishing House.

16. Shike M (1996) Nutrition therapy for the cancer patients. Hematol Oncol Clin North Am 10, 221-234.

17. Rivadeneira DE, Evoy D \& Fahey TJ (1998) Nutritional support of the cancer patient. Cancer J Clin 48, 69-80.

18. Capra S, Bauer J \& Davidson W (2002) Nutritional therapy for cancer-induced weight loss. Nutr Clin Prac 17, $210-213$. 